Trauma Berufskrankh 2017 · [Suppl 1]: 19:S7-S11 DOI 10.1007/s10039-016-0206-x

Online publiziert: 28. September 2016

๑) Springer-Verlag Berlin Heidelberg 2016

CrossMark

\section{Grundgelenke der Zehen}

\section{Großzehengrundgelenk}

Ständige axiale Stöße oder immer wiederkehrende Translationsbewegungen wie bei Fußballspielern oder auch Balletttänzern führen zum Verschleiß des Knorpels der gelenkbildenden Anteile des Großzehengrundgelenkes (• Abb. 1).

In Frühstadien der Arthrose ist oft ein Gelenkerhalt möglich. Es bieten sich dann Operationsverfahren nach Valenti ([1]; - Abb. 2a und b) oder aber Osteotomien zur Verkürzung und Plantarisierung nach Youngswick [2] oder Waterman-Green [2] an.

Wegen der meist schon fortgeschrittenen Arthrose ist eine Arthrodese des Grundgelenkes oft sinnvoller (• Abb. 3).

Die Einstellung der Arthrodese in allen Ebenen entscheidet über den Erfolg der Operation. In der Transversalebene

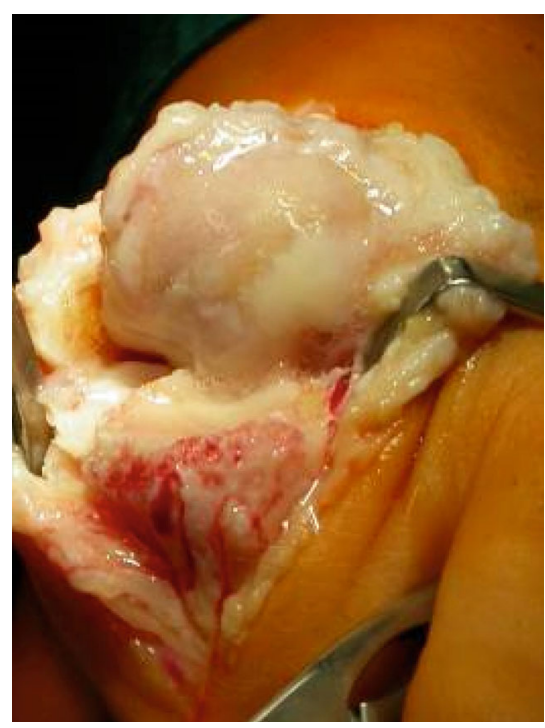

Abb. 1 ム Zerstörter Gelenkknorpel bei einem Fußballspieler

\title{
Kai Olms
}

Chirurgische Praxis Bad Schwartau, Bad Schwartau, Deutschland

\section{Posttraumatische Arthrosen am Fuß}

ist eine Valgusposition von $10^{\circ}$ anzustreben, in der Sagittalebene sollte die Zehenbeere den Boden leicht berühren, was etwa einer Dorsalextension von $10^{\circ}$ entspricht. Die Stellung ist intraoperativ auf einer festen Unterlage zu prüfen.

In Fehlstellung eingestellte Arthrodesen bedürfen der Achskorrektur (- Abb. 4a und b). Die in der Regel $\mathrm{zu}$ weit in Dorsalextension eingestellte Arthrodese wird durch eine öffnende Keilosteotomie mit Apex im Bereich der ehemaligen Gelenklinie korrigiert.

\section{Grundgelenk der 2. Zehe}

Das Grundgelenk der 2. Zehe unterliegt hohen Belastungen insbesondere bei Sprintern und Springern. Kompressions- und Translationskräfte führen zu Knorpelschäden, die von einem Morbus Köhler-Freiberg abzugrenzen sind. Das Anbohren des meist zentral gelegenen Knorpeldefektes führt die Sportler nach einer Rekonvaleszenz von bis zu 6 Monaten zurück zur sportlichen Leistung (• Abb. 5 und 6).

Die Fallzahlen sind allerdings gering, und ein Vergleich zu Umstellungsosteotomien fehlt. Eine Versteifung dieses Ge- lenkes wird nicht empfohlen. Als Lösung bei Versagern bietet sich die Operation nach Stainsby an ([3]; - Abb. 7), die allerdings bei Sportlern wegen der dann fehlenden Abstoßkraft nicht zu empfehlen ist.

\section{Lisfranc-Gelenkreihe (Tarso- metatarsal [TMT]-1- bis -5- Gelenke)}

Die Verletzungen der Lisfranc-Gelenkreihe sind in Diagnostik und Therapie immer wieder eine Herausforderung. Schwere Verletzungen werden in der Regel nicht übersehen und nach den geltenden Prinzipien versorgt. Probleme bereiten die Niedrigrasanzverletzungen, die oft als Mittelfußdistorsion beschrieben werden, aber ein pathognomonisches Hämatom aufweisen (• Abb. 8).

Nicht übersehen werden dürfen der knöcherne Bandausriss des Lisfranc-Ligamentes (• Abb. 9) und die begleitende "Nussknackerverletzung“ des Os cuboideum (• Abb. 10), weil sich hieraus Behandlungskonsequenzen ableiten.

Sowohl unbehandelte als auch korrekt osteosynthetisch versorgte Verletzungen
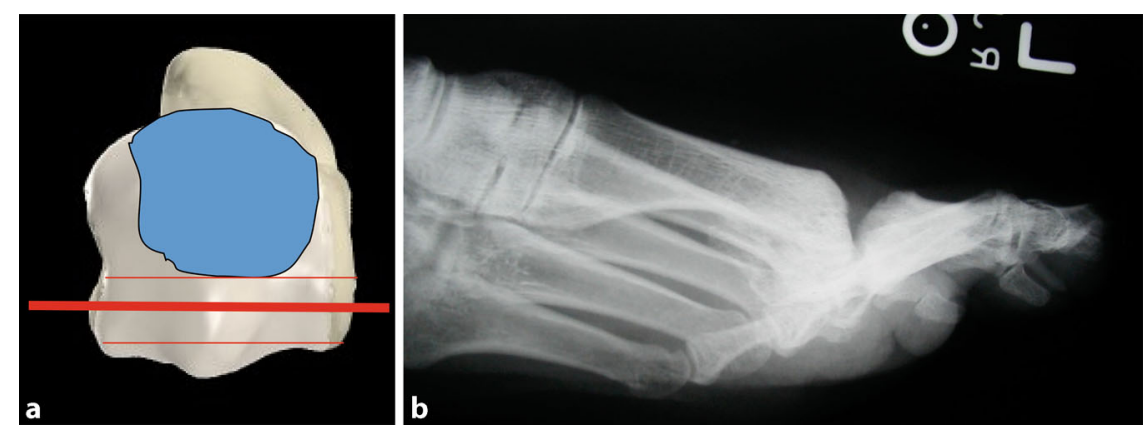

Abb. $2 \Delta$ a Die breite Linie markiert die Resektionslinie zwischen der Untergrenze des Knorpeldefektes (blau) und den Sesambeinfurchen. b Postoperatives Röntgenbild 


Übersichten

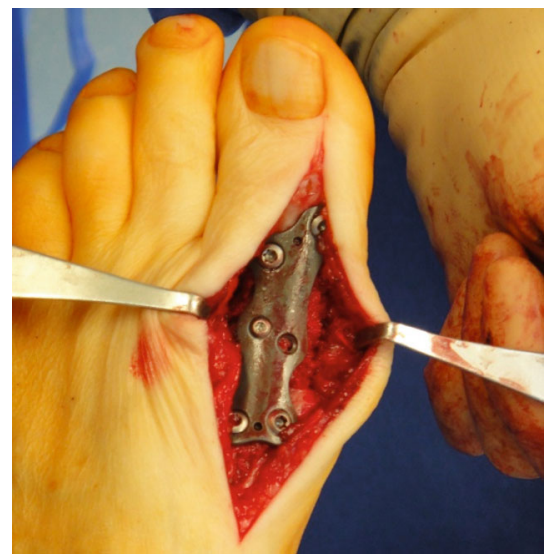

Abb. $3 \Delta$ Großzehengrundgelenkarthrodese mit Beckenkammspaninterposition

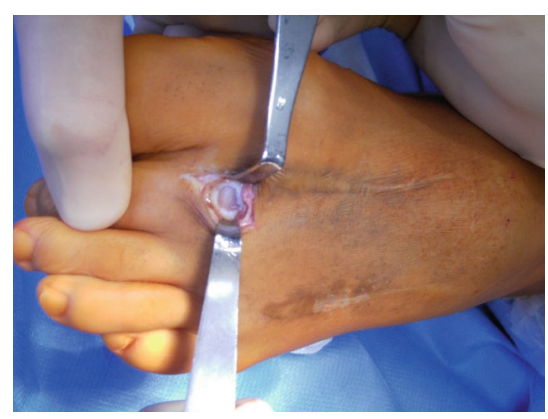

Abb. 5 ム Zentraler Knorpeldefekt Metatarsale (MT) 2 bei einem Weitspringer

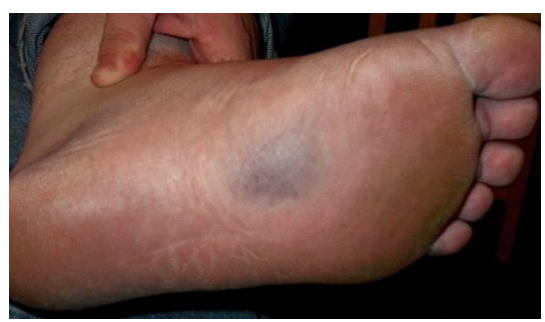

Abb. 8 × Pathognomonisches Hämatom der Verletzung des Lisfranc-Gelenkes
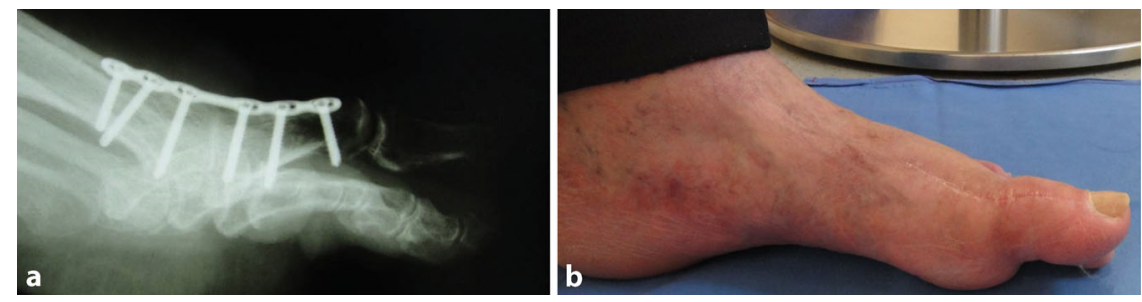

Abb. $4 \Delta$ a In zu starker Dorsalextension eingestellte Arthrodese des Großzehengrundgelenkes. b Klinisches Bild der funktionslosen Großzehe

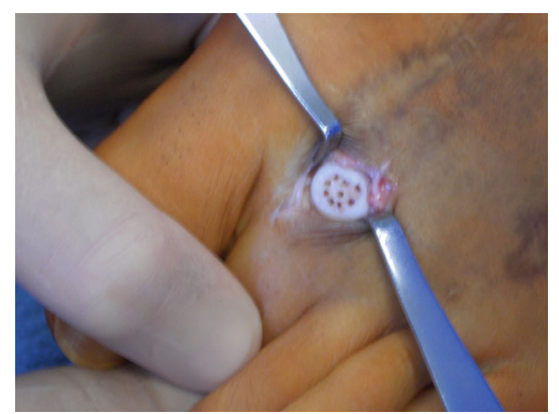

Abb. $6 \Delta$ Nach Anbohren des Defektes

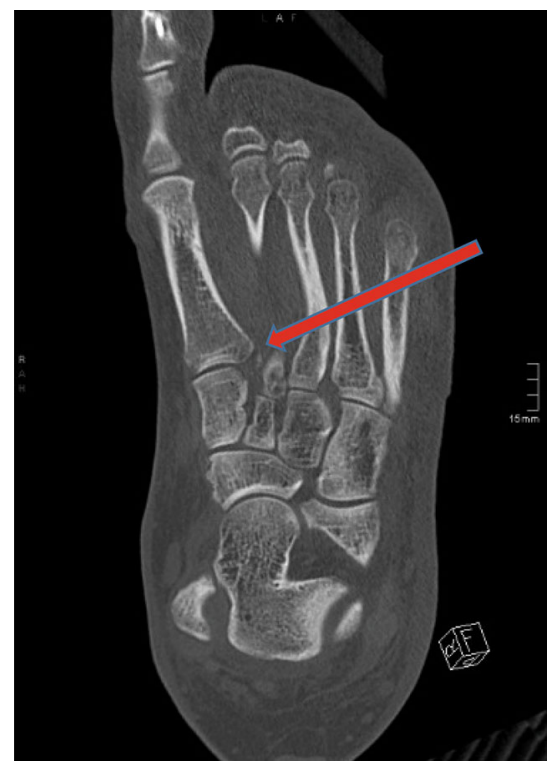

Abb. $9 \Delta$ Knöcherner Ausriss des Lisfranc-Ligamentes (Fleck-Zeichen - Pfeilmarkierung)

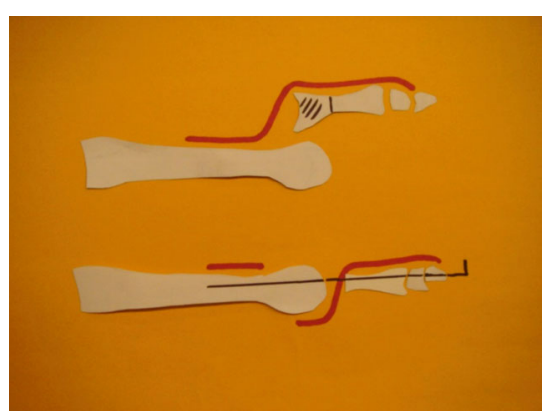

Abb. 7 A Operation nach Stainsby. Basisresektion des Grundgliedes mit Interposition der langen Strecksehne

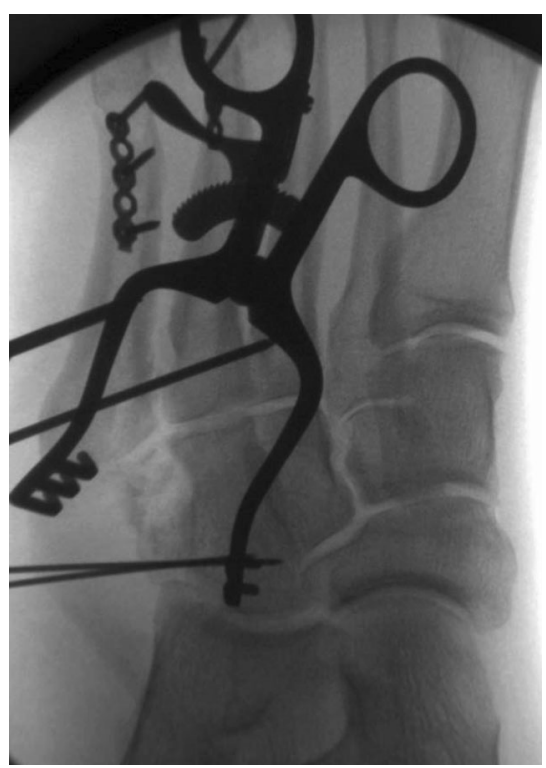

Abb. $10 \Delta$ "Nussknackerverletzung" des Os cuboideum 


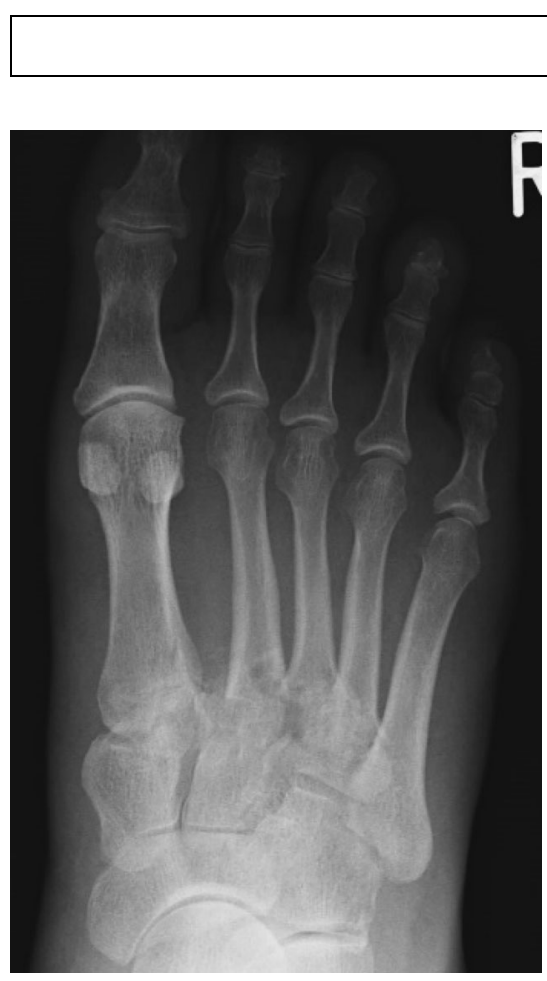

Abb. 11 A Homolaterale Lisfranc-Luxation

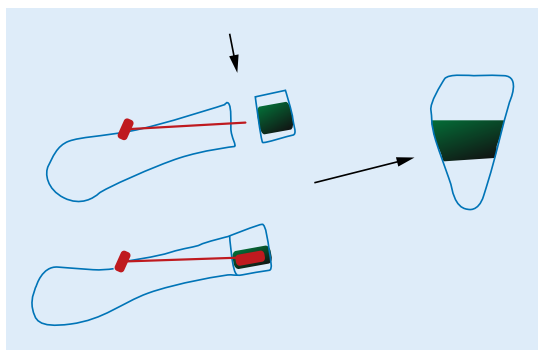

Abb. 13 ॥ Einbringen des Führungsdrahtes für die Schraube unter Sicht auf die Zielregion im Cuneiforme

haben ein hohes Potenzial an posttraumatischen Arthrosen.

In der Primärversorgung der Verletzung kann gelenkerhaltend operiert werden. Hierzu eignen sich temporäre Arthrodese und Distanzarthrodesen (- Abb. 11 und 12).

In übersehenen Verletzungen und auch bei neuropathischen Füßen ist die primäre endgültige Arthrodese das sinnvollste Verfahren. Die Versteifung der durch Computertomographie (CT) verifizierten befallenen Gelenke bietet die einzige Behandlungsmöglichkeit der medialen und zentralen Gelenke. Die lateral gelegenen Gelenke sollten möglichst nicht versteift werden, um die Mobilität der lateralen Säule zu erhalten.

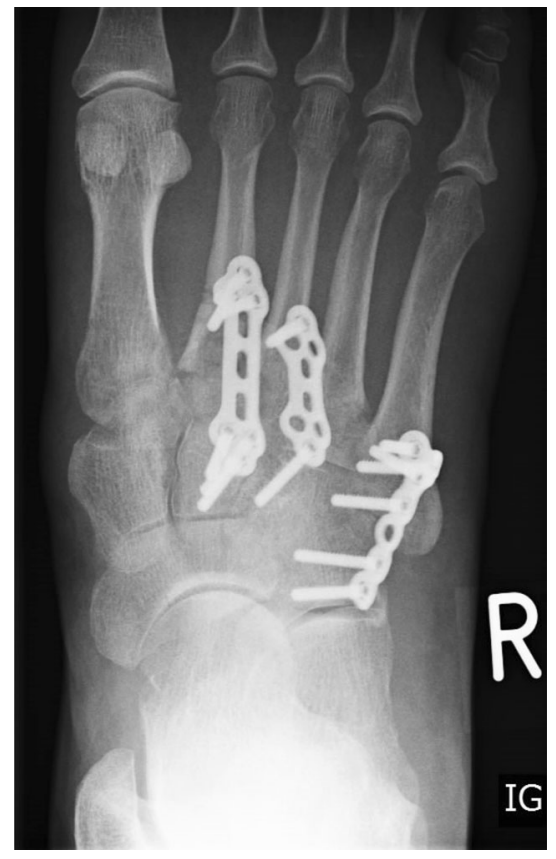

Abb. $12 \Delta$ Distanzarthrodese des Lisfranc-Gelenkes

Die Versteifung eines einzelnen Gelenkes der Reihe erfordert die Interposition eines trikortikalen Beckenkammspans. Das TMT 2 hat die geringste sagittale Beweglichkeit und einen großen $\mathrm{He}$ belarm und neigt deshalb gehäuft zur isolierten - auch nichttraumatischen - Arthrose [4]. Die Fixation kann unter Sicht erfolgen, sodass die Positionierung der Zugschraube erleichtert wird (• Abb. 13).

Alternativ bieten sich verschiedene Plattensysteme oder Staples zur stabilen Fixation der Arthrodesen an.

\section{Naviculocuneiforme Gelenkreihe (NC-1- bis -3- Gelenke)}

Die Verletzung und damit auch die posttraumatische Arthrose dieser Gelenke sind selten. Operativ bietet sich hier die Versteifung an. Zur Differenzierung der Schmerzen sollte wegen der anatomischen Nähe verschiedener Gelenke eine Probeinfiltration mit einem Lokalanästhetikum erfolgen. Werden NC 1 und 2 versteift, ist die Arthrodese NC 3 nicht erforderlich (• Abb. 14).
Trauma Berufskrankh 2017 - [Suppl 1]: 19:S7-S11

DOI 10.1007/s10039-016-0206-x

(c) Springer-Verlag Berlin Heidelberg 2016

\section{K. Olms}

\section{Posttraumatische Arthrosen am Fuß}

\section{Zusammenfassung}

Nach intraartikulären Verletzungen kann es auch bei optimaler operativer oder konservativer Versorgung zu posttraumatischen Arthrosen kommen. Im Bereich des Vor-, Mittel- und Rückfußes ist die Arthrodese meist die einzige Möglichkeit der schmerzbefreienden Rekonstruktion. Die Arthrodesen müssen in funktionsgerechter Stellung durchgeführt werden. In-situ-Arthrodesen sind häufig ausreichend. Interpositionsarthrodesen und Spongiosaplastiken dienen der Kompensation von Substanzverlusten oder nicht akzeptablen Verkürzungen.

Schlüsselwörter

Arthrodese - Trauma - Rekonstruktion . Funktion $\cdot$ Schmerz

\section{Posttraumatic arthritis of the foot}

Abstract

Intra-articular injuries may lead to posttraumatic arthritic conditions even after perfect surgical anatomical reconstruction or conservative treatment. In the regions of the forefoot, midfoot and hindfoot arthrodesis of the affected joints is frequently the only possibility to alleviate the pain. The position of the fusion should follow the functional requirements. In situ fusion is sufficient in most cases. Interpositional tricortical bone grafts or cancellous bone grafts may be necessary to compensate for loss of substance or unacceptable reduction in length.

\section{Keywords}

Arthrodesis - Trauma - Reconstruction .

Function · Pain 


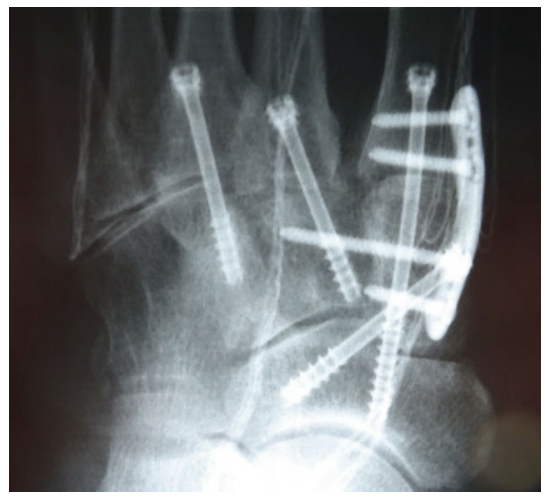

Abb. $14 \Delta$ Versteifung des naviculocuneiformen Gelenkes

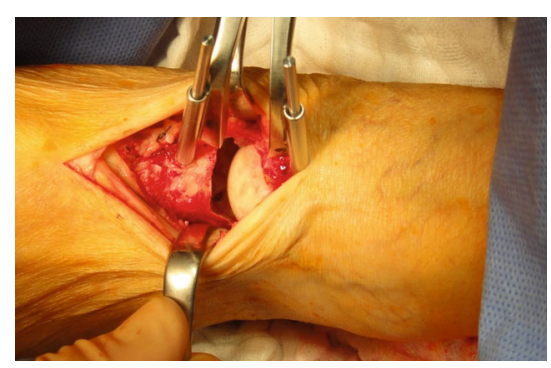

Abb. $16 \Delta$ Darstellung des talonavikularen Gelenkes über einen dorsomedialen Zugang

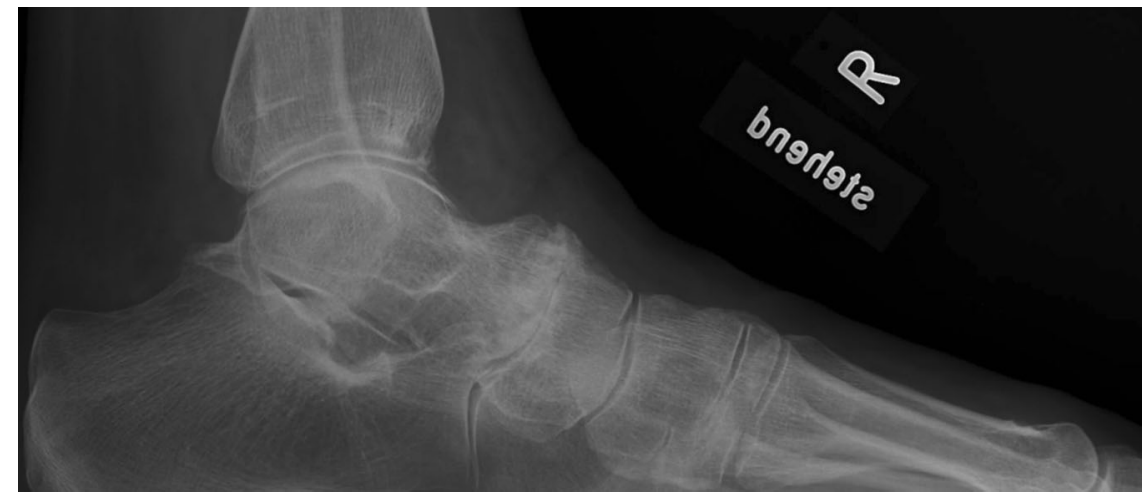

Abb. 15 ॥ Symptomatische Arthrose des talonavikularen Gelenkes

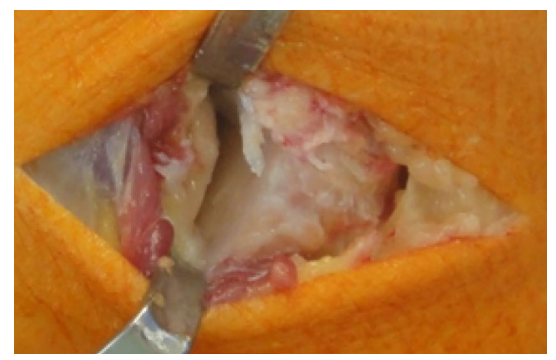

Abb. 17 ॥ Arthrose im Kalkaneokuboidgelenk - Kalkaneus rechts

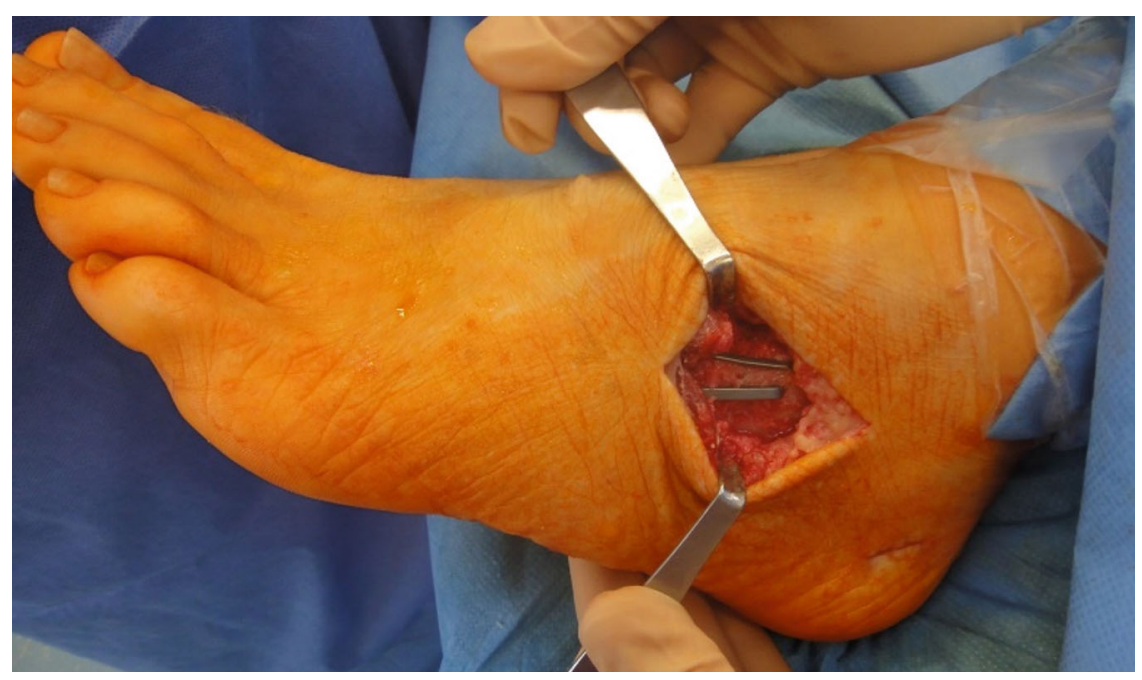

Abb. 18 ム Kalkaneokuboidarthrodese mit Fixation durch Staples

\section{Chopart-Gelenk}

Die Verletzung des Chopart-Gelenkes stellt eine schwerwiegende Verletzung einer wichtigen Gelenkreihe dar [5]. Frühzeitig erkannte Verletzungen können meist gelenkerhaltend operiert werden. Übersehene Verletzungen oder aber spät erkannte Verletzungen bedürfen der Arthrodese.

\section{Talonavikulares Gelenk}

Dieses biomechanisch wichtige Gelenk kann bei einer symptomatischen Arthrose nur versteift werden (• Abb. 15).

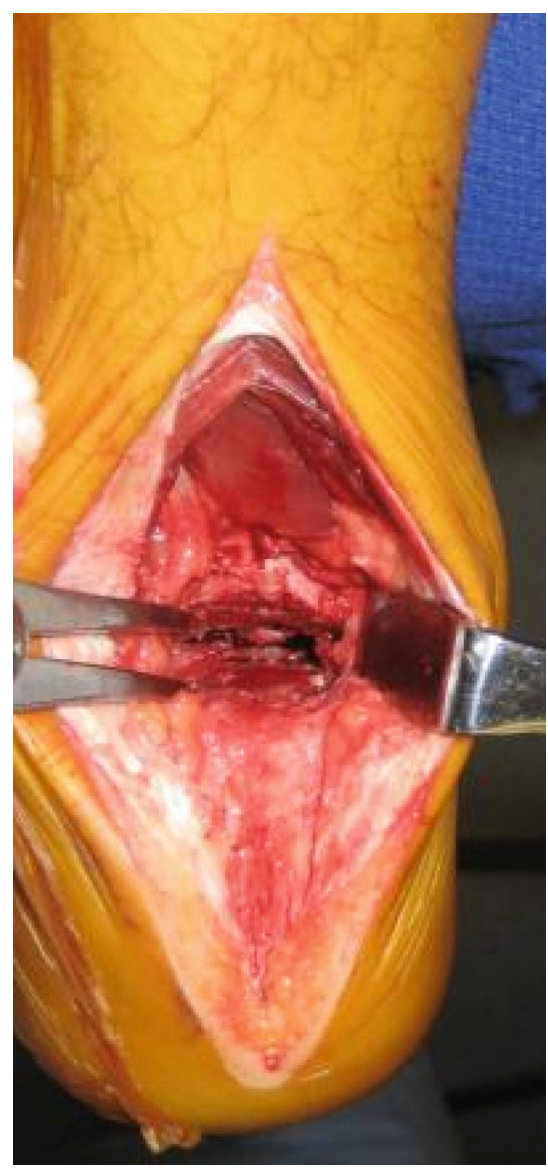

Abb. 19 ॥ Posteriorer Zugang zur subtalaren Arthrodese

Arthrosen entstehen gehäuft durch Avaskuläre Nekrose (AVN) des Os naviculare und durch Talusfrakturen in dessen Kopfbereich.

Über einen dorsomedialen Zugang zwischen der Sehne des Extensor Hallucis longus (EHL) und Tibialis anterior (TA) wird das gesamte talonavikulare (TN) Gelenk übersichtlich dargestellt. 


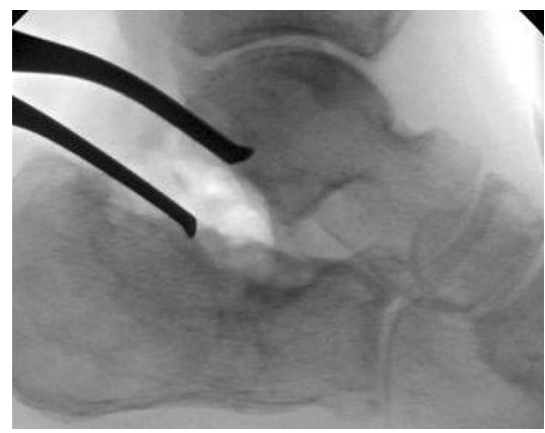

Abb. $20 \Delta$ Aufspreizen des subtalaren Gelenkes

Die Vorbereitung der Arthrodese umfasst neben der Entknorpelung auch die Entfernung der subchondralen Lamelle, um eine möglichst primäre Arthrodese zu erreichen (• Abb. 16).

Die plantigrade Einstellung der Arthrodese ist wichtig und sollte in allen Ebenen erfolgen. Die Fixation kann unter Sicht erfolgen und vorzugsweise mit 2 bis 3 kanülierten Doppelgewindeschrauben und ggf. in Kombination mit einer Platte durchgeführt werden.

Eine postoperative Entlastung ist meist für 6 bis 8 Wochen erforderlich.

\section{Kalkaneokuboidgelenk}

Die Arthrose des Kalkaneokuboid (CC)Gelenkes ist meist folge einer Fraktur des Processus anterior calcanei, des Os cuboideum oder einer Instabilität des $\mathrm{Ge}$ lenkes durch Verletzung des Lig. calcaneocuboidale und/oder des Lig. bifurcatum. Die Arthrodese kann direkt oder mit Interposition eines trikortikalen Beckenkammspans erfolgen. Die Stabilisierung erfolgt vorzugsweise durch Staples, aber auch durch eine Schrauben- oder Plattenosteosynthese (• Abb. 17 und 18).

\section{Talonavikular- und Kalkaneo- kuboidarthrodese}

In Ausnahmefällen ist die Arthrodese des Chopart-Gelenkes erforderlich. Diese sollte erfolgen, wenn auf der medialen Seite ein größerer Substanzverlust vorliegt. Die Anschlussarthrose des CC-Gelenkes ist dann vermeidbar [5].

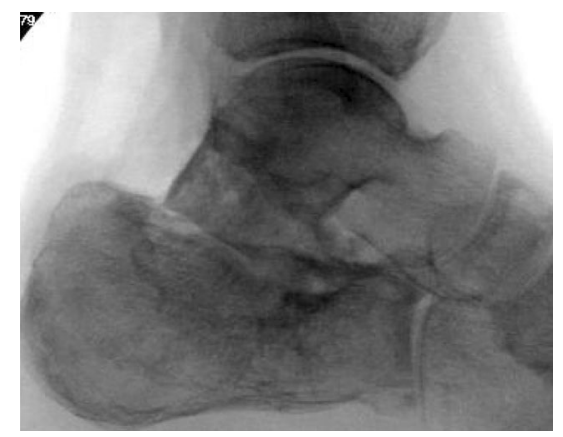

Abb. 21 ॥ Implantierter Beckenkammspan

\section{Subtalargelenk}

Die posttraumatische Arthrose des Subtalargelenkes (STG) ist eine häufig beobachtete Komplikation nach intraartikulären Talusfrakturen oder Fersenbeinbrüchen. Neben den Talushalsfrakturen, die je nach Verletzungstyp in einem hohen Prozentsatz zu posttraumatischen Arthrosen neigen, können auch periphere Talusfrakturen zu einem Kollaps des STG führen.

Bei entsprechender Beschwerdesymptomatik ist die Arthrodese des STG erforderlich. Die Arthrodese kann als In-situ-Arthrodese oder aber als achskorrigierende Arthrodese durchgeführt werden. Die Distraktionsarthrodese mit einem von posterior eingebrachten Beckenkammspan ist nur bei anteriorem Impingement indiziert und bringt sonst keine besseren Ergebnisse als die In-situArthrodese ([6]; - Abb. 19, 20 und 21).

\section{Fazit für die Praxis}

- Auch bei perfektem operativem oder konservativem Management von intraartikulären Verletzungen sind posttraumatische Arthrosen nicht sicher zu vermeiden.

- Die Arthrodese der befallenen Gelenke bietet eine operative Maßnahme mit gut reproduzierbaren Ergebnissen und guter Aussicht auf Schmerzfreiheit bei erhaltener Funktion des Fußes.

- Entscheidend für ein gutes Ergebnis einer Arthrodese ist deren Positionierung in Funktionsstellung des jeweiligen Gelenkes.
- Jedes Gelenk des Fußes kann verletzt werden und folgerichtig eine posttraumatische Arthrose entwickeln.

\section{Korrespondenzadresse}

Dr. K. Olms
Chirurgische Praxis Bad
Schwartau
Am Kurpark 1, 23611 Bad
Schwartau, Deutschland
Olms@chirurgie-bad-
schwartau.de

\section{Einhaltung ethischer Richtlinien}

Interessenkonflikt. K. Olms gibt an, dass kein Interessenkonflikt besteht.

Dieser Beitrag beinhaltet keine vom Autor durchgeführten Studien an Menschen oder Tieren.

The supplement containing this article is not sponsored by industry.

\section{Literatur}

1. Olms K, Grady JF, Schulz AP (2008) Die Resektionsarthroplastik nach Valenti zur Behandlung des fortgeschrittenen Hallux rigidus. Oper Orthop Traumatol 20:492-499. doi:10.1007/s00064-0081505-6

2. Co AY, Chang TJ, Camasta CA (2013) In: McGlamry ED (Hrsg) Comprehensive textbook of foot and ankle surgery, 4. Aufl. Wolters Kluwer/Lippincott Williams \& Wilkins, Philadelphia, S343-361

3. Stainby GD (1997) Pathological anatomy and dynamic effect of the displaced plantar plate and the importance of the integrityof the plantar platedeep transverse metatarsal tie-bar. Ann R Coll Surg Engl 79(1):58-68

4. Hardcastle $\mathrm{PH}$, Reschauer R, Kutscha-Lissberg E, Schoffmann W (1982) Injuries to the tarsometatarsal joint. Incidence, classification and treatment. JBone Joint Surg Br 64:349-356

5. Zwipp H (1994) Chirurgie des Fußes. Springer, Wien, S131-146

6. Chandler JT, Bonar SK, Anderson RB, Davis H (1999) Results of in situ subtalar arthrodesis for late sequelae of calcaneus fractures. Foot Ankle Int 20(1):18-24 